\title{
Histological Study of Age-Related Changes of Internal Thoracic Artery - A Cross-Sectional Study from Calicut, India
}

\author{
Anagha S. ${ }^{1}$, Usha Devi K.B. ${ }^{2}$ \\ ${ }^{1}$ Department of Anatomy, Government Medical College, Calicut, Kerala, India. ${ }^{2}$ Department of Anatomy, \\ Government T.D. Medical College, Alappuzha, Kerala, India.
}

\section{ABSTRACT}

\section{BACKGROUND}

Internal thoracic artery (ITA) is the ideal graft material for coronary artery bypass grafting (CABG). It's structural characteristics and resistance to atherosclerotic changes make it ideal for this. It is in this scenario that we attempted to study the histological characteristics and age-related changes.

\section{METHODS}

This cross-sectional study was done on stained slides of segments of ITAs obtained from 100 fresh cadavers during autopsy. Age range was between $20-80$ years. The segments were collected at various rib levels constituting proximal, middle and distal segments. The wall parameters were measured and recorded. The specimens were grouped according to age into six groups with intervals of 10 years and analysis of variance (ANOVA) test were conducted on the samples using Statistical Package for Social Science (SPSS) software.

\section{RESULTS}

The mean thickness of tunica intima in the proximal segment of 100 specimens was $26.792 \mu \mathrm{m}$, tunica media was $171.437 \mu \mathrm{m}$ and the thickness increased significantly with age with $(\mathrm{F}=33.038, \mathrm{P}=0.00),(\mathrm{F}=87.910, \mathrm{P}=0.00)$ respectively. In one specimen, the intimal thickening increased to $96.325 \mu \mathrm{m}$. The mean thickness of tunica intima in the middle segment was $19.665 \mu \mathrm{m}$, tunica media was $172.302 \mu \mathrm{m}$ and it increased significantly with age with $(\mathrm{F}=71.885, \mathrm{P}=0.00),(\mathrm{F}=85.481, \mathrm{P}=$ $0.00)$ respectively. The mean thickness of tunica intima in the distal segment was $18.157 \mu \mathrm{m}$, tunica media was $146.278 \mu \mathrm{m}$ and it increased significantly with age ( $\mathrm{F}$ $=58.847, \mathrm{P}=0.00),(\mathrm{F}=66.137, \mathrm{P}=0.00)$ respectively. The intima media ratio (IMR) increased significantly with age. The IMR of proximal segment of $2 \%$ specimens were found to be greater than 0.25 indicating atherosclerosis. The vessel wall parameters decreased from proximal to distal segments.

\section{CONCLUSIONS}

The vessel wall thickness and IMR was found to increase with age. The incidence of atherosclerosis was $2 \%$ and in old age. It is clear that the ITA is the ideal graft for CABG in younger individuals. An understanding of these attributes of ITA will be helpful to the cardiothoracic surgeons. Moreover, the results of this study throw light on the vessel wall changes in the population of present times. Further clinical study is needed to assess the effect of other risk factors.

\section{KEY WORDS}

Internal Thoracic Artery, Tunica Intima, Tunica Media, Intima Media Ratio, Atherosclerosis
Corresponding Author:

Dr. Usha Devi K.B.,

Professor and $H O D$

Department of Anatomy,

Government T. D. Medical College,

Alappuzha, Kerala, India.

E-mail: ushakbdevi1@gmail.com

DOI: $10.14260 / j e m d s / 2021 / 613$

How to Cite This Article:

Anagha S, Usha Devi KB. Histological study of age-related changes of internal thoracic artery - a cross-sectional study from Calicut, India. J Evolution Med Dent Sci 2021;10(35):2999-3004, DOI: 10.14260/jemds/2021/613

Submission 01-06-2021, Peer Review 23-07-2021, Acceptance 30-07-2021, Published 30-08-2021.

Copyright (c) 2021 Anagha S. et al. This is an open access article distributed under Creative Commons Attribution License [Attribution 4.0 International (CC BY 4.0)] 


\section{BACKGROUND}

Kay et al. (1976) studied ITA segments from routine postmortem examinations \& these segments were evaluated microscopically. Significant atherosclerotic narrowing was seen in $4.2 \% .^{1}$

Singh (1983) reported that atherosclerosis was present in only three out of one hundred and fifty patients $(2 \%)$ who underwent bilateral internal thoracic arteriography. ITA atherosclerosis was found either proximally or distally. He came to a conclusion that atherosclerotic involvement of the ITA is rare which makes it a suitable vessel for coronary bypass. $^{2}$

Acar et al. (1991) studied the wall thickness of internal thoracic artery and radial arteries comparatively and observed the following. Architectural differences exist between the media of both arteries. The ratio of the thickness of media to thickness of intima was reported to be higher in the internal thoracic artery compared to the radial artery ( 4 and 3 respectively). ${ }^{3}$

The ratio of intima to media thickness (R) was used as an index for arteriosclerosis for ITAs and left anterior descending arteries (LADs) by Kobayashi et al. (1993). Grades I to IV were given based on the value of $\mathrm{R}$. The $\mathrm{R}$ of the ITAs was roughly $1 / 10$ of that of LADs. A low arteriosclerotic grade was shown by most ITAs, without any variation in grade along their length and a low $\mathrm{R}$ in all segments. ${ }^{4}$

Wharton et al. (1994) observed that intimal changes of ITAs were minimal and no significant occlusive disease was present in persons dying of atherosclerosis-related causes. The media thickness decreased from proximal to distal. ${ }^{5}$

Cizek et al. (2007) conducted a study on paired internal thoracic arteries obtained from 89 autopsies. Atherosclerotic lesions were identified in 19 autopsies. Significant correlation was found with atherosclerotic lesion development above 75 years. In the remaining cases without atherosclerosis, significant association with pre-atherosclerotic intimal thickening was seen only in age group $51-75$ years. Age appears to be a constant risk factor in development of preatherosclerotic intimal hyperplasia after the 4th decade. These observations point to the fact that in some contexts, intimal hyperplasia is possibly a part of atherosclerosis, and its development be influenced by traditional risk factors for atherosclerosis. ${ }^{6}$

Puri et al. (2010) concluded that the prevalence of atherosclerosis in ITA was similar to what had been seen in other studies all over the world. ${ }^{7}$

Borovic et al. (2013) concluded that the grade of atherosclerosis increases gradually with aging as revealed by morphometric analysis. The increase in thickness of medial layer suggests the potential for positive remodelling of the ITA during aging and atherosclerosis. ${ }^{8}$

Morphometric analysis by Fonseca et al. (2019) included intimal width (IW), medial width (MW) and intima/media ratio (IMR) as the parameters. He did not observe any overt atherosclerotic lesions in his study. Age was found to be the main clinical predictor for intimal hyperplasia. ${ }^{9}$

\section{Aims \& Objectives}

To describe the changes in tunica intima thickness and any signs of atherosclerosis, tunica media thickness and intima media ratio with advancing age.

\section{METHODS}

This is a cross-sectional study conducted from December 2018 to December 2020. Sections of internal thoracic artery were taken and age-related changes in its histological appearance was noted. 100 specimens were collected and observation was made. Specimens were collected from Department of Forensic Medicine, Government Medical College, Calicut.

\section{Inclusion Criteria}

Internal thoracic artery collected from cadavers in the age groups 20 - 80 years.

\section{Exclusion Criteria}

Grossly damaged arteries.

\section{Methodology}

After removal of sternum with ribs, internal thoracic artery was dissected at various rib levels. Three bits one each from proximal, middle \& distal portions were taken. The bits after harvesting were immediately transferred to small labelled bottles containing fixative solution. The fixative solution was buffered neutral formalin. The arterial samples were obtained within 12 to 24 hours post death.

Specimen was kept in fixative for 3 days. Dehydration was carried out by placing it in consecutively increasing strength of alcohol solution. Clearing of the dehydrated specimens were done with xylene followed by molten paraffin impregnation and paraffin wax embedding. Sections were cut using microtome. Transverse sections of the artery bits were taken from a block at a thickness of 5 micrometre. After incubation, the sections were stained using various stains. Stains used were haematoxylin \& eosin stain (H \& E stain), Verhoeff van Gieson stain, Orcein stain.

Rehydration was done in graded alcohol and thereafter deparaffinized sections were rinsed with distilled water and subsequently stained. Finally, the sections were dehydrated with absolute alcohol, cleared with xylene and cover slip applied after using DPX as the mounting medium.

The stained slides were read with the help of trinocular microscope and a software called Zen. Manual scaling was done. The tunica intima thickness was measured from the periphery of lumen to the internal elastic lamina. Separate measurements for proximal, middle and distal segments were taken. Tunica media was measured from internal elastic lamina to external elastic lamina. Within the tunica media, elastic coat thickness and muscle coat thickness were measured separately. The measurements were taken from various sites in the same slide radially and average was taken for reporting. This was done for proximal, middle and distal segments of each artery. 
Intima media ratio was also determined to predict the atherosclerotic changes. The grade of atherosclerosis was determined with the help of intima media ratio as proposed by Kobayashi and colleagues: grade 0 - intima-to-media ratio $\leq 0.25$; grade 1 - intima-to-media ratio $>0.25$ but $\leq 0.5$; grade 2 - intima-to-media ratio $>0.5$ but $\leq 0.75$; grade 3 - intimato-media ratio $>0.75$. If the lesion was diffuse, grade was increased by one, indicating that the intimal thickness included more than $50 \%$ of the cross-sectional circumference. Complete obliteration by thickening or thrombosis was given a grade of 4 . For each specimen, the grade was derived from that section with the most advanced observed disease. ${ }^{4}$

\section{Statistical Analysis}

With the above data a master chart was prepared and statistical analysis was done using SPSS software. ANOVA was used for statistical analysis. A P value of less than 0.05 was considered significant.

\section{RESULTS}

100 internal thoracic artery specimens of age groups varying from 20 - 80 years were studied under light microscope and divided into six groups with interval of 10 years each.

The histological characteristics were carefully studied in each group and compared with those of other groups. H \& E stain and special stains were used for microscopic sections.

\section{Group-I}

Tunica intima thickness ranged from 11.359 to 18.966 micrometre in the proximal segment. Tunica intima thickness of middle segment changed from 8.632 to 14.528 and of distal segment from 6.387 to 12.544 . The values do not show uniform increase and varied in between.

Tunica media thickness showed uniform increase from 90.568 to 142.364 micrometre in proximal, 96.342 to 136.235 in middle and 77.367 to 120.361 micrometre in distal segments.

Intima media ratio increased as age advanced in proximal (from 0.079 to 0.194 ), middle (from 0.073 to 0.108 ) and distal segments (from 0.072 to 0.120 ) though there are minor variations.

No evidence of atherosclerosis was noted in group 1

\section{Group-II}

Tunica intima thickness varied from 17.632 to 24.967 micrometre in the proximal segment. Tunica intima thickness of middle segment changed from 8.362 to 18.864 and of distal segment from 11.368 to 16.975 micrometre.

Tunica media thickness increased from 116.643 to 164.325 micrometre in proximal, 137.756 to 162.568 in middle and 116.638 to 155.362 micrometre in distal segments.

Intima media ratio increased as age advances in proximal, middle and distal segments though there were minor individual variations.
No evidence of atherosclerosis was noted in group II.

\section{Group-III}

Tunica intima thickness varied from 14.536 to 27.965 micrometre in the proximal segment. Tunica intima thickness of middle segment changed from 14.432 to 29.356 and of distal segment from 14.532 to 22.914 . The values did not show uniform increase and varied in between.

Tunica media thickness increased from 152.326 to 223.566 micrometre in proximal, 132.546 to 164.569 in middle and 106.923 to 154.362 micrometre in distal segments.

Intima media ratio increased as age advanced in proximal, middle and distal segments though there were minor individual variations.

No evidence of atherosclerosis was noted in group III.

\section{Group-IV}

Tunica intima thickness varied from 16.784 to 32.588 micrometre in the proximal segment. Tunica intima thickness of middle segment changes from 10.564 to 25.368 and of distal segment from 11.658 to 26.354 . The values did not show uniform increase and varied in between.

Tunica media thickness showed increase from 116.359 to 204.565 micrometre in proximal, 97.826 to 312.867 in middle and 77.456 to 166.321 micrometre in distal segments.

Intima media ratio increased as age advanced in proximal, middle and distal segments though the individual variations were minor. Largest value of intima media ratio in this group was 0.255 .

No evidence of overt atherosclerosis was noted in group IV. Grade 1 change was present in a 59-year-old with a moderately increased intima media ratio.

\section{Group-V}

Tunica intima thickness varied from 30.548 to 96.325 micrometre in the proximal segment. Tunica intima thickness of middle segment changed from 8.723 to 40.166 and of distal segment from 9.866 to 32.242 .

Tunica media thickness increased from 133.568 to 260.258 micrometre in proximal, 154.265 to 302.514 in middle and 96.188 to 243.864 micrometre in distal segments.

Intima media ratio increased as age advanced in proximal, middle and distal segments though there were minor individual variations. There was evidence of atherosclerosis in group- $V$, that is, grade 1 change in a single specimen. Intima media ratio of that specimen was 0.430 .

\section{Group-VI}

Tunica intima thickness varied from 39.268 to 48.600 micrometre in the proximal segment. Tunica intima thickness of middle segment changed from 35.537 to 42.561 and of distal segment from 21.168 to 36.528 .

Tunica media thickness showed increase from 246.837 to 311.545 micrometre in proximal, 268.472 to 402.415 in middle and 199.567 to 256.357 micrometre in distal segments. 
Intima media ratio increased as age advanced in proximal, middle and distal segments though the individual variations were there. No evidence of atherosclerosis was noted in group-VI.

\begin{tabular}{|c|c|c|c|c|c|c|c|c|c|c|}
\hline \multirow{2}{*}{ Age } & \multirow{2}{*}{ Frequency } & \multicolumn{3}{|c|}{ Tunica Intima $(\mu \mathrm{m})$} & \multicolumn{3}{|c|}{ Tunica Media $(\mu \mathrm{m})$} & \multicolumn{3}{|c|}{ Intima Media Ratio } \\
\hline & & $\mathbf{P}^{*}$ & $\mathbf{M}^{* *}$ & $\mathrm{D}^{* * *}$ & $\mathbf{P}^{*}$ & $\mathbf{M}^{* *}$ & $\mathrm{D}^{* * *}$ & $\mathbf{P}^{*}$ & $\mathbf{M}^{* *}$ & $\mathbf{D}^{* * *}$ \\
\hline $20-30$ & 22 & 15.903 & 10.243 & 9.283 & 114.965 & 109.844 & 98.099 & 0.141 & 0.093 & 0.949 \\
\hline $30-40$ & 19 & 21.316 & 14.717 & 14.465 & 148.136 & 147.564 & 128.899 & 0.144 & 0.098 & 0.112 \\
\hline $40-50$ & 12 & 24.882 & 19.259 & 17.925 & 163.357 & 153.271 & 140.749 & 0.155 & 0.106 & 0.127 \\
\hline $50-60$ & 22 & 28.567 & 20.795 & 21.292 & 170.525 & 171.095 & 151.111 & 0.169 & 0.125 & 0.131 \\
\hline $60-70$ & 17 & 38.45 & 27.025 & 24.285 & 224.499 & 211.206 & 186.156 & 0.174 & 0.129 & 0.132 \\
\hline $70-80$ & 8 & 42.957 & 39.192 & 30.035 & 283.955 & 352.013 & 230.309 & 0.186 & 0.132 & 0.136 \\
\hline \multicolumn{11}{|c|}{ Table 1. Mean Values of Tunica Intima, Media and Intima Media Ratio in Various Age Groups } \\
\hline
\end{tabular}
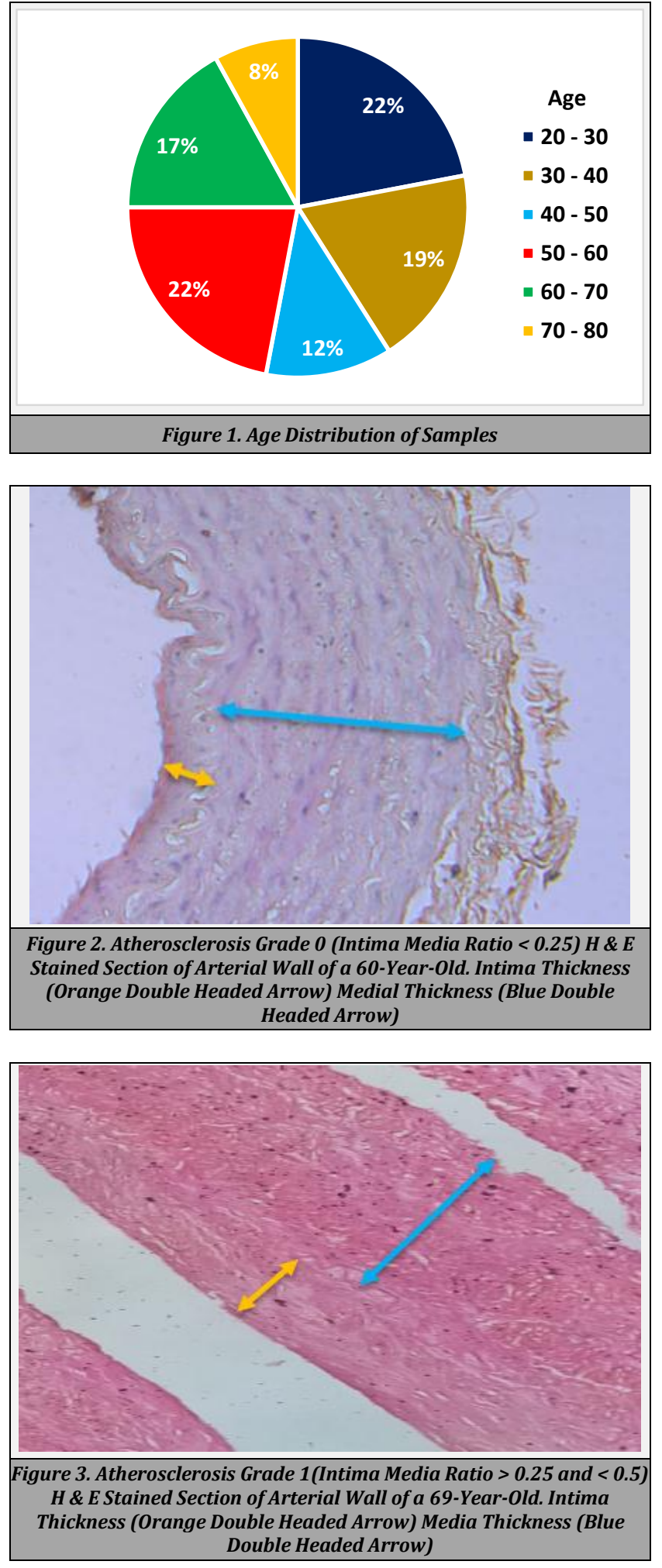

\begin{tabular}{|cccc|}
\hline & & F & P Value \\
Tunica intima & Proximal & 33.038 & 0.000 \\
thickness & Middle & 71.885 & 0.000 \\
& Distal & 58.847 & 0.000 \\
Tunica media & Proximal & 87.910 & 0.000 \\
thickness & Middle & 85.481 & 0.000 \\
& Distal & 66.137 & 0.000 \\
Intima media ratio & Proximal & 3.723 & 0.004 \\
& Middle & 7.966 & 0.000 \\
& Distal & 12.122 & 0.000 \\
\hline Table 2. Variations within Sample Means (F) and P Values \\
\multicolumn{3}{c}{ (Statistically Significant if P < 0.005) } \\
\hline
\end{tabular}

\section{DISCUSSION}

\section{Changes in Tunica Intima with Age}

In the present study, thickness of tunica intima was found to increase gradually with age which was statistically relevant. The tunica intima thickness in the proximal segment of 20 30 age group ranged from $11.359-18.966 \mu \mathrm{m}$. In $30-40$ group it varied from 17.632 to $24.967 \mu \mathrm{m}$. Range in $40-50$ group - 14.536 to 27.965 , in 50 - 60 group 16.784 to 32.588 , in $60-70$ group -30.548 to 96.325 , in $70-80$ group 39.268 to 48.600 . In 60 - 70 group, proximal segment showed an abnormal increase to $96.325 \mu \mathrm{m}$ in a single specimen aged 69.

Tunica intima thickness of middle and distal segments also showed a gradual increase in in all groups. The mean thickness of tunica intima in middle segment was $10.243 \mu \mathrm{m}$ in 20 - 30 age group, $14.717 \mu \mathrm{m}$ in $30-40,19.259$ in $40-50$, 20.795 in $50-60,27.025$ in $60-70$ and 39.192 in $70-80$ age group. The results of the study are consistent with the findings of Kneubil et al. and also with Borovic et al. The thickness of the intimal layer was greater in the proximal segments as reported by Kneubil et al. ${ }^{10}$ Borovic et al. stated that intima thickness increases significantly with aging. ${ }^{8}$

According to Reddy et al. ${ }^{11}$ the thickness of the tunica intima was found to be constant in all age groups which is in contrast to the present study. In this study, it significantly increased with age. Nakajima et al. reported that there was no intimal thickening in the internal thoracic arteries. ${ }^{12}$ In the current study, intimal thickening was seen in 2 percentage of specimens in the age groups of $50-60$ and $60-70$.

The increase in the tunica intima thickness may be a part of intimal hyperplasia, a precursor of atherosclerotic changes. The incidence of overt atherosclerotic changes is minimal in internal thoracic artery as described in the present study. 


\section{Changes in Tunica Media with Age}

In this study, thickness of tunica media also increases with age. The tunica media thickness which was around 114.965 $\mu \mathrm{m}$ at 25 years increased to $283.955 \mu \mathrm{m}$ at 75 years. Similar gradation was obtained in middle \& distal segments also.

The increase in thickness in tunica media in the present study may be due to proliferation of vascular smooth muscle cells.

\section{Intima Media Ratio}

In the present study, intima media ratio increased significantly with age. The intima media ratio is intimal thickness divided by medial thickness. The intima media ratio is a measure of arteriosclerosis of internal thoracic artery. Two specimens had intima media ratio greater than 0.25 . Grade 1 arteriosclerotic change were observed in proximal segments of 2 specimens. The grade of atherosclerosis was determined with the help of intima media ratio as proposed by Kobayashi and colleagues: grade 0 - intima-to-media ratio $\leq 0.25$; grade 1 - intima-to-media ratio $>0.25$ but $\leq 0.5$; grade 2 - intima-to-media ratio $>0.5$ but $\leq 0.75$; grade 3 - intima-to-media ratio $>0.75$. If the lesion was diffuse, grade was increased by one, indicating that the intimal thickness included more than $50 \%$ of the cross-sectional circumference. Complete obliteration by thickening or thrombosis was given a grade of 4.4

The results of the present study were corroborative with Borovic et al. in that the intima-to-media ratio increased with age. $^{8}$

It was inferred from the current study that the increase in intimal thickness is more than that of medial thickness in atherosclerosis.

\section{Atherosclerosis}

In the present study, overt atherosclerotic lesions were absent. But sections from the proximal part of two ITA specimens out of the 100 showed grade 1 arteriosclerotic changes (aged $59 \& 69$ ). The incidence of atherosclerosis was only $2 \%$. Although luminal narrowing (blockage in the lumen of artery due to increase in intimal thickness) was present in specimens showing atherosclerotic changes, it was not more than $50 \%$.

Kay's (1976) study showed atherosclerotic narrowing in $4.2 \%$ of ITAs. ${ }^{1}$

Singh (1983) reported atherosclerosis in $3 \%$ of ITAs and the lesions in the ITAs were found either close to the origin or around the terminal bifurcation. ${ }^{2}$ In this study atherosclerosis was present in specimens close to the origin. Wharton et al. claimed that no artery showed significant occlusive disease, even from persons dying of atherosclerosis-related causes. ${ }^{5}$ No atherosclerotic lesions were seen in the internal thoracic arteries by Nakajima et al.12 Only $2 \%$ of specimens showed atherosclerotic changes in the present study. This evidence points to the fact that atherosclerosis is minimal in ITA.

In the present study, the frequency of atherosclerosis was $4.54 \%$ in sixth decade and it increased to $5.88 \%$ in seventh decade with overall incidence of $2 \%$. Puri et al.'s finding of 6 $\%$ prevalence of atherosclerosis is slightly higher than the present study. ${ }^{7}$
In the view of Cizek et al. pre-atherosclerotic intimal hyperplasia routinely may be part of the disease process of atherosclerosis, and that its formation may be influenced by traditional risk factors for atherosclerosis. ${ }^{6}$

According to Reddy et al. (2011) special micro-anatomical features of the ITA wall may protect it from age related pathological changes. Elastic nature of the media of ITA may be associated with lower incidence of atherosclerosis or intimal hyperplasia in samples even in elderly cases. ${ }^{11}$

\section{CONCLUSIONS}

There was a significant increase in the tunica intima \& tunica media thickness with aging. The intima media ratio also significantly increased with age. Overt atherosclerotic lesions were absent in the vessel wall. The incidence of atherosclerosis was $2 \%$ and in old age. It is clear that the ITA is the ideal graft for CABG in younger individuals. An understanding of these attributes of ITA will be helpful to the cardiothoracic surgeons. Moreover, the results of this study throw light on the vessel wall changes in the population of present times.

\section{Limitations of This Study}

Further clinical studies is needed to assess the effect of other risk factors like diabetes, hypertension etc.

Data sharing statement provided by the authors is available with the full text of this article at jemds.com.

Financial or other competing interests: None.

Disclosure forms provided by the authors are available with the full text of this article at jemds.com.

\section{REFERENCES}

[1] Kay HR, Korns ME, Flemma RJ, et al. Atherosclerosis of the internal mammary artery. The Annals of Thoracic Surgery 1976;21(6):504-7.

[2] Singh RN. Atherosclerosis and the internal mammary arteries. Cardiovasc Intervent Radiol 1983;6(2):72-7.

[3] Acar C, Jebara V, Portoghese M, et al. Comparative anatomy and histology of the radial artery and the internal thoracic artery. Implication for coronary artery bypass. Surgical and Radiologic Anatomy 1991;13(4):283-8.

[4] Kobayashi H, Kitamura S, Kawachi K, et al. A pathohistological and biochemical study of arteriosclerosis in the internal thoracic artery, a vessel commonly used as a graft in coronary artery bypass surgery. Surg Today 1993;23(8):697-703.

[5] Wharton SB, Cary NRB, Gresham GA. Observations on detailed histology of the internal thoracic artery and their relevance to its comparatively low incidence of atheroma. Clinical Anatomy 1994;7(4):215-8.

[6] Cizek SM, Bedri S, Talusan P, et al. Risk factors for atherosclerosis and the development of preatherosclerotic intimal hyperplasia. Cardiovasc Pathol 2007;16(6):344-50. 
[7] Puri N, Gupta PK, Sharma J, et al. Prevalence of atherosclerosis in coronary artery and internal thoracic artery and its correlation in North-West Indians. Indian Journal of Thoracic and Cardiovascular Surgery 2010;26:243-6.

[8] Borović ML, Borović S, Marinković-Erić J, et al. A comprehensive morphometric analysis of the internal thoracic artery with emphasis on age, gender and left-toright specific differences. Histol Histopathol 2013;28(10):1299-314.

[9] Fonseca DA, Antunes PE, Antunes MJ, et al. Histomorphometric analysis of the human internal thoracic artery and relationship with cardiovascular risk factors. PLoS One 2019;14(1):e0211421.
[10] Kneubil MC, Gomes WJ, Aquino MS, et al. Sequential histomorphometric study of the left internal thoracic artery. Brazilian Journal of Cardiovascular Surgery 2006;21(4):371-6.

[11] Reddy S, Kumar P, Prasad K. Histomorphometric and sympathetic innervation of the human internal thoracic artery. Clinics (Sao Paulo) 2011;66(1):131-6.

[12] Nakajima T, Tachibana $K$, Takagi $N$, et al. Histomorphologic superiority of internal thoracic arteries over right gastroepiploic arteries for coronary bypass. The Journal of Thoracic and Cardiovascular Surgery 2016;151(6):1704-8. 